\title{
ÉDITORIAL
}

\author{
*
}

\section{DU MONDE DES EAUX \\ DANS LA VIE DES HOMMES}

\author{
par Maurice FONTAINE \\ Membre de l'Académie des Sciences et de l'Académie de Médecine
}

$\boldsymbol{I}^{\mathrm{s}}$

EST SUR QUE L'EAU fut longtemps, pour les scientifiques, comme pour les poìtes, le symbole de l'unité. Considérée d'abord comme un élément, puis comme une combinaison d'hydrogène et d'oxygène, il était légitime de regarder l'eau comme une unité chimique parfaitement définie alors que l'existence des isotopes était insoupçonnée. Mais aujourd'hui que nous connaissons la présence, dans les eaux, de trois isotopes d'hydrogène, le protium ${ }^{1} \mathrm{H}$, le deutérium ${ }^{2} \mathrm{H}$ ou $\mathrm{D}$, le tritium ${ }^{3} \mathrm{H}$ ou $\mathrm{T}$, de trois isotopes d'oxygène, ${ }^{16} \mathrm{O},{ }^{17} \mathrm{O}$ et ${ }^{18} \mathrm{O}$, il est sûr que, ces isotopes se trouvant en des proportions variables dans les échantillons d'eau prélevés en divers points de l'hydrosphère, il n'y a pas une eau, mais des eaux. Certains auteurs négligent le tritium, qui se trouve, en effet, à des concentrations extrêmement faibles dans notre hydrosphère, de l'ordre de $10^{-15}$ à $10^{-16}$ de l'hydrogène total. Cependant, cette concentration a pu s'élever dans l'hydrogène de l'eau de pluie, lors des essais thermonucléaires aériens des années 1963-1964, jusqu'à 10-12. Mais il existe dans la nature, en proportions non négligeables, quatre molécules différentes d'eau : $\mathrm{H}_{2}{ }^{16} \mathrm{O}$, la plus légère et de beaucoup la plus abondante, $\mathrm{HD}_{2}{ }^{18} \mathrm{O}, \mathrm{H}_{2}{ }^{17} \mathrm{O}$ et $\mathrm{H}_{2}{ }^{18} \mathrm{O}$. Sans doute, ces quatre variétés d'eau ne se trouvent jamais isolées dans l'hydrosphère, mais du fait que les trois eaux les plus lourdes se trouvent en proportions variables dans la variété d'eau la plus légère, il existe une grande diversité d'eaux du point de vue de leur composition isotopique. Cette composition peut d'ailleurs se modifier rapidement, du fait des phénomènes d'évaporation et de condensation. La volatilité de l'eau légère étant un peu plus élevée que celle des molécules plus lourdes, l'évaporation a pour effet d'enrichir une eau en isotopes lourds, alors que la vapeur engendrée est plus pauvre en ces éléments. Le phénomène est évidemment inverse pour la condensation, l'eau de condensation étant généralement plus riche en isotopes lourds que la vapeur dont elle provient.

De ces phénomènes découlent les faits suivants :

10) Les eaux de mer sont généralement plus riches en isotopes denses que les eaux douces continentales.

$2^{\circ}$ ) Les nuages formés par l'évaporation océanique dans les régions chaudes ou tempérées du globe s'appauvrissent progressivement en itosopes lourds au fur et à mesure qu'ils donnent lieu à des précipitations au cours de leur progression vers les régions de plus en plus froides. Il en résulte que les eaux de précipitation sont plus

Notre Bureau a le privilège de bénéficier de l'autorisation de publier en priorité la Conférence que le Professeur FonTanne, ancien Directeur du Muséum d'Histoire Naturelle et de I'Institut Océanographique, a prononcée le lundi 19 septembre dernier devant Mme le Ministre de la Santé au cours de la séance solennelle d'ouverture des Journées Pharmaceutiques Internationales de Paris 1977. 


\section{ÉDITORIAL}

légères en climat froid qu'en climat chaud. Elles sont donc de plus en plus légères à mesure que la latitude croît. Dans une même région, elles sont plus légères en montagne qu'en plaine. En un même lieu, elles sont plus légères en saison froide qu'en saison chaude.

Ainsi donc, il existe, en ce qui concerne la composition isotopique, une grande variété et une grande variabilité des eaux dans la nature.

La connaissance de cette variété dans la composition isotopique a-t-elle des conséquences en sciences pharmaceutiques? Sans aucun doute. Quand, par exemple, on exploite une eau minérale, la connaissance de l'extension de l'aire d'alimentation de la nappe exploitée est essentielle pour la définition des périmètres de protection de cette nappe contre les pollutions de surface, ainsi que pour l'estimation du taux de renouvellement du système. La détermination de la composition isotopique de l'eau minérale d'une part, des précipitations dans la région, en fonction notamment de la température moyenne et donc de l'altitude d'autre part, de diverses eaux infiltrées et collectées enfin, permet de répondre à la question primordiale pour les responsables d'une eau minérale : l'origine des eaux de la nappe exploitée. De plus, la composition isotopique de $\mathrm{H}_{2} \mathrm{O}$ peut ne pas être sans conséquence sur ses activités biologiques. On a montré notamment que la substitution de $\mathrm{D}_{2} \mathrm{O}$ à $\mathrm{H}_{2} \mathrm{O}$ modifie l'action de l'eau sur certains paramètres de la vie, la fonction de reproduction, chez la souris, étant la première touchée.

Mais les eaux parfaitement pures, déjà variées par les rapports isotopiques qui les caractérisent, n'existent pratiquement pas dans notre hydrosphère. Les eaux naturelles dans l'air ou dans la terre se trouvent en contact avec un si grand nombre d'éléments ou de corps solubles qu'elles constituent une innombrable variété d'eaux. On distingue, parmi nos eaux dites douces, des eaux plus ou moins dures, des eaux bicarbonatées, séléniteuses, ferrugineuses, sulfatées, sulfurées et beaucoup d'autres. Les eaux marines ne sont pas moins variées : alors que leur salinité peut atteindre $42 \%$ os dans la mer Rouge, elle peut s'abalsser à moins de $4 \%$ dans la Baltique. En un même point la concentration en certains métaux peut varier largement selon le moment de l'année. Les eaux ne contiennent pas seulement des minéraux et des métaux, mais aussi des matières organiques, à des concentrations très variables selon le lieu, et, en même masse d'eau, selon l'époque et selon les conditions climatiques. C'est grâce à cet ensemble, c'est grâce à cette soupe primitive, comme on a nommé ce mélange aqueux très complexe, qu'a pu naître la vie. Le monde des eaux a bien été, en effet, selon toute vraisemblance, le berceau initial de cette manifestation surprenante : la vie. Au cours d'une longue évolution est apparu l'Homme. Qu'en est-il aujourd'hui du Monde des Eaux dans la Vie des Hommes ? Tel est le thème de l'Introduction à ces journées.

Soulignons d'abord que l'homme, à l'état de nature, sans bagage scientifique, avec ses seuls organes des sens et son bon sens, a parfaitement conscience qu'il est en présence d'une grande variété d'eaux, du fait de leurs couleurs, de leurs transparences, de leurs formes, de leurs saveurs, de leur dynamique, de leur fluidité, de leur langage. Des eaux claires de nos ruisseaux de montagne aux eaux foncées de Rio Negro, des eaux limpides et céruléennes des Caraỉbes à certaines eaux rouges tropicales, que de nuances. L'ail admire aussi non seulement les couleurs, mais les formes innombrables des eaux, «l'élusive architecture des cascades» selon l'expression de Roger Caillois, celle plus figée des stalactites ou celle des icebergs.

Les saveurs des eaux douces comme celles des eaux marines sont extrêmement variées et les responsables de la qualité des eaux qui nous sont distribuées disposent de "goûteurs d'eau " à la sensibilité gustative longuement exercée et particulièrement subtile. Ce sujet sera traité par $M$. MonTIEL. La dynamique des eaux est aussi extrêmement variable. Certaines eaux de source et de torrent jaillissent impétueusement, alors que celles des fleuves au cours lent déroulent leur capricieux ruban avec un calme tel que, vues d'une certaine altitude, elles paraissent figées. Les eaux marines peuvent présenter la surface lisse d'un miroir, un léger frémissement sous la caresse d'une brise légère, le clapotis sous le fouet d'un alizé ou le chaos d'une tempête.

Les langages des eaux sont très divers. Qui de nous n'a tendu l'oreille au murmure d'un ruisseau, ne s'est pris à rêver au bruissement régulier de la marée de morte eau qui vient, vaguelette après vaguelette et par temps calme, s'étendre en un soupir discret sur une longue plage déserte, ne s'est senti stimulé par l'éclat joyeux de la vague qui se brise, ne fut empoigné par le grondement d'un cyclone. Les hommes ne peuvent donc douter qu'ils se trouvent en présence d'un monde des eaux aussi varié que le monde des hommes. Il n'est pas jusqu'au toucher qui ne puisse apporter aux familiers des eaux des informations sur leur diversité. Naviguant à l'aviron, dans ma jeunesse, avec un vieux marin sur un modeste canot, il nous arrivait souvent, pour reprendre force et courage, de plonger la main dans l'eau. Mon compagnon n'immergeait pas seulement sa main ouverte, il prenait l'eau à poignée, la palpait et il lui arrivait, m'incitant à l'imiter, de me dire : «Sentez comme la mer est grasse ici aujourd'hui, elle lampera ce soir ». Et c'était vrai. La nuit venue, c'était une pluie de feu qui coulait de chaque pale d'aviron émergée. La sensation perçue au toucher était celle qui résultait de l'existence dans les eaux, de substances huileuses à la surface de la mer, substances qui ont été maintenant décelées et analysées dans de nombreuses zones marines et résultant de la présence dans les eaux d'un certain plancton comportant des éléments luminescents responsables de ce phénomène qu'on appelle souvent la phosphorescence de la mer, mais qui est, en fait, une bioluminescence. Ainsi, un vieux marin, accoutumé de palper les eaux, en 


\section{ÉDITORIAL}

connaissait la diversité bien avant que les chimistes océanographes n'aient mis en évidence la grande diversité des eaux en ce qui concerne leur teneur en organismes planctoniques luminescents et riches en lipides, leur grande diversité aussi en ce qui concerne leur richesse en acides gras, en triglycérides et en cérides. entourent.

Tous les organes des sens sont donc là pour nous convaincre de la grande variété des eaux qui nous

Ces eaux, quelle est leur importance dans la vie des Hommes? Rappelons d'abord qu'elle est considérable dans la vie en général et qu'elle en conditionne de nombreuses manifestations. La grande majorité des cellules sont constituées pour la plus grande part d'eau, si bien que l'on a pu écrire qu'elles étaient d'abord et avant tout de l'eau animée. Ces cellules pour la plupart ne sont pas isolées, mais vivent dans un milieu aqueux.

L'eau environne donc la cellule comme elle la constitue en majeure partie. Qu'elle conditionne les manifestations vitales, cela est particulièrement évident dans le cas de ces semences végétales, de ces spores de bactéries et de champignons, de ces oufs de certains Crustacés, le fameux Artemia salina notamment, des larves de certains insectes, de nombreux petits animaux eux-mêmes, les nématodes, les rotifères, les tardigrades qui peuvent subsister pendant plusieurs années, après une dessiccation très poussée. Mais alors, cependant que subsiste la vie, la plupart de leurs manifestations vitales sont pratiquement suspendues. Celles-ci reprennent dès qu'on leur apporte l'eau en quantité suffisante.

L'Homme cependant n'a pas cette faculté. Sa vie même est étroitement et constamment hydrodépendante et ses mécanismes physiologiques exigent impérativement, d'une façon presque continue, un apport d'eau. D'ailleurs la physiologie comparée nous apprend que l'eau est d'autant plus nécessaire, d'autant plus précieuse que l'animal est plus évolué. Encore que le manque d'expérimentation rigoureuse - et nous nous en félicitons ne nous permette pas d'avancer de valeur précise, il semble bien que l'Homme ne puisse supporter sans succomber, une perte d'eau de plus de $20 \%$, alors que la souris résiste jusqu'à une perte de $34 \%$, la grenouille jusqu'à $50 \%$, certains escargots désertiques jusqu'à $77 \%$, une sangsue jusqu'à $92 \%$. Et quand «l'importune vieillesse " nous annonce notre fin prochaine, c'est que l'eau nous quitte, les tissus perdant leur aptitude à fixer l'eau. Mais je n'insiste pas sur ces aspects des corrélations entre l'eau et la physiologie de l'Homme puisque ce sujet doit étre traité demain par le Professeur Legrain. Ce que je dois souligner ici, c'est le point suivant : quand j'ai proposé pour cette introduction le titre "Du Monde des eaux dans la Vie des Hommes ", $j$ 'ai voulu exprimer deux idées : d'abord que les eaux étaient très variées, je crois l'avoir rappelé, et ensuite, que tous les hommes n'avaient pas les mêmes besoins en eau et que tous les hommes n'avaient pas la même conscience de la valeur des eaux.

Les hommes d'une même population n'ont pas les mêmes besoins en eau selon leurs activités, musculaires et professionnelles, selon les conditions du milieu qui les entoure, selon leurs adaptations physiologiques, physiques et comportementales. Nous savons tous que selon la température et le degré d'hygrométrie du milieu dans lequel nous nous trouvons, selon l'importance et la nature du travail que nous y accomplissons, nos besoins d'eau sont différents. Nous savons tous aussi que l'homme adapté à un certain exercice dans un climat donné peut se comporter très différemment de l'homme non accoutumé et subitement confronté à ces conditions. Le Professeur Bernard METz a relaté une étude comparative faite au Sahara avec un groupe de militaires du contingent et un groupe d'autochtones d'une tribu des environs d'Hassi-Messaoud, les uns et les autres étant exposés aux mêmes ambiances dans les mêmes conditions. Or, Metz constatait, chez les autochtones et chez ceux-ci seulement, une répulsion à ingérer la moindre quantité d'eau pendant les deux premières heures d'un travail pourtant relativement dur, dans une exposition à très haute température. C'est seulement après avoir contracté un déficit hydrique de $3 \grave{a} 4 p .100$ du poids du corps qu'ils acceptaient de commencer à boire. Si on les forçait à boire, dès le début de l'exposition, en fonction des pertes d'eau calculées, ils présentaient des crampes, des syncopes assez comparables à celles observées dans certaines intoxications par l'eau. Tout se passe, chez ces Hommes, comme si le mécanisme de la soif ne se déclenchait qu'avec un certain retard, un retard toutefois qui ne traduit pas une insuffisance de la régulation hydrique de l'organisme, mais au contraire un avantage en ce qui concerne l'épargne de l'eau. L'organisme n'accepte en effet de boire qu'à partir du moment où il atteint un degré de déshydratation partielle qui assure un freinage suffisant de l'élimination rénale et donc un régime économique du métabolisme hydrique. Au début d'une exposition à la chaleur, laisser s'installer dans l'organisme un certain état de déshydratation limitée est sans doute un moven de réduire les prises d'eau par la suite.

Mon confrère T. MonoD a décrit par quel entraînement et quel effort de volonté il était parvenu à faire une longue marche dans le Sahara avec une moyenne d'eau ingérée de 1041 cc par jour. Pendant plusieurs jours, la prise est descendue au-dessous d'un litre, alors que pour un Européen, en climat tempéré, la consommation moyenne de l'adulte est évaluée à environ $2.500 \mathrm{cc}$. La différence apparaît encore plus impressionnante si l'on songe que l'explorateur saharien se liorait à de fortes étapes quotidiennes en plein vent et en plein soleil et que, dans ces conditions, un Européen non entraîné aurait dî̀ absorber plusieurs litres d'eau. $\mathcal{N}$ 'ayant ni les mêmes disponibilités, ni les mêmes besoins en eau, tous les hommes n'ont pas la même conscience de la valeur de l'eau. J'en donnerai quelques exemples mais voudrais d'abord rappeler que nombreux sont nos sages ancêtres 


\section{ÉDITORIAL}

qui ont bien ressenti l'importance de l'eau. Environ 4 siècles avant 7.-C., Platon écrivait : l'eau est la chose la plus nécessaire à l'entretien de la vie, mais ajoutait-il, il est aisé de la corrompre. "Loué soit mon Seigneur, écrivait St François d'Assise, pour seur eau qui est si utile, humble, précieuse et chaste ». Mais actuellement qu'en est-il ? Des efforts si efficaces ont été accomplis pour fournir à tous dans nos cités une eau potable qu'un nutritionniste aussi éminent qu'André MAYER s'exprimait ainsi, en 1953, dans le discours qu'il prononçait sous la Coupole : «Impérieusement, inexorablement, nos besoins nous rappellent qu'après peu de minutes quand l'air nous manque, peu de jours quand c'est l'eau, peu de semaines quand c'est la nourriture, nous sommes en danger de mort. L'air nous est donné ; et là où nous avons décidé de vivre, l'eau est à portée de la main. Choisir, se procurer la nourriture est autrement difficile ». "L'eau est à portée de la main » disait André MAYER. J'ai beaucoup d'admiration pour la vie et l'auvre d'André MAYER, mais je dois cependant souligner que c'est là une affirmation d'un citadin qui n'a qu'un robinet à tourner pour obtenir de l'eau potable ou d'un alpiniste qui a toujours à sa disposition une eau naturelle propre, sous forme de liquide, de neige ou de glace. André MAYER était l'un et l'autre, et ceci explique son propos. Mais pour les hommes qui ont voyagé à travers le monde ou tout simplement dans certaines campagnes de nos régions, l'eau consommable n'est pas toujours à portée de notre main. Fe me rappelle, au cours de mon enfance, et pendant certains étés, avoir fait la queue avec les autres habitants du village pour obtenir un peu d'eau de l'unique fontaine, qui n'était autorisée à débiter son précieux liquide qu'à un certain moment de la journée. Je me souviens d'avoir vécu dans une commune où l'eau disponible dans chaque maison n'était que l'eau de pluie recueillie dans une citerne. Quand celle-ci était à sec, nous attendions le porteur d'eau — qui devait aller chercher celle-ci à plusieurs hilomètres -- comme le Messie. Quand j'étais à Port-Etienne, un navire devait se rendre aux îles Canaries pour nous approvisionner en eau ; celle-ci n'était donc pas à portée de la main.

La situation a évolué, me direz-vous. C'est exact. Les conditions d'approvisionnement en eau se sont améliorées dans de nombreuses régions, mais non partout à travers le monde. De plus, la qualité des eaux potables définie par les normes de Bruxelles, encore que celles-ci apparaissent à certains hygiénistes insuffisamment rigoureuses, est de plus en plus difficile à respecter, même en France, du fait que les sources de pollution se multiplient, que les pollutions elles-mêmes se diversifient avec le développement industriel qui rejette sans cesse dans la nature des produits nouveaux dont l'inocuité n'est pas toujours assurée et dont l'élimination par les divers procédés d'épuration, quand ceux-ci peuvent être mis en auvre, n'est pas toujours certaine. Que l'eau consommable ne soit pas toujours à portée de la main des hommes, j'en veux pour preuve le fait que cette année même, inaugurant la conférence internationale sur l'eau de Mar del Plata, Luis Jauregui, Ministre des ressources hydrauliques de l'Argentine et Président de la conférence, soulignait combien, pour une grande partie du tiers monde, le manque d'eau a un aspect actuellement même dramatique. On peut estimer, déclarait-il, que chaque jour 25.000 personnes meurent de maladies consécutives au manque d'eau. Selon d'autres estimations, la moitié au moins des populations rurales du globe ne dispose pas d'eau saine et un tiers au moins des habitants des villes se trouve dans la même situation. De tous ces faits, les hommes de nos régions n'ont pas suffisamment conscience et ils considèrent l'eau comme un fluide sans grande valeur, qui ne mérite guère l'attention et les soins que nous voulons ici lui porter.

En voici un exemple plus récent. En 1965, dans l'un de nos plus grands quotidiens, dont le titre évoque un célèbre écrivain français du $18^{\mathrm{e}}$ siècle, paraissait un éditorial intitulé "Que d'eau, que d'eau », dans lequel l'auteur écrivait ceci : "L'une des phrases les plus atroces que l'on puisse écrire est que notre corps est composé pour $71 \%$ d'eau. Elle dit, poursuivait l'auteur, notre pauvreté, notre faiblesse et la précarité de notre destin ». Etant à cette époque Président de l'Association nationale pour la Protection des Eaux, je pensais être qualifié pour répondre que l'eau n'était indice ni de pauvreté, ni de faiblesse et j'apportais à ce point de vue des arguments qui me semblaient de valeur. Mon papier, je ne le vis jamais paraitre ; il partit sans doute à la corbeille, rejoindre tous ceux qui auraient pu susciter chez le lecteur quelques réflexions sur des affirmations contestables. Et pourtant que d'exemples! Que d'hommes bien portants meurent chaque jour par manque d'eau, que de malades succombent encore, alors qu'un ou deuri litres de soluté salé auraient pu les sauver.

A cette même époque, je me rendais au Ministère de l'Education nationale, afin de proposer le canevas de quelques leçons destinées aux enfants des écoles pour leur apprendre la valeur des eaux propres et les inciter à éviter tout ce qui peut les souiller. Ces canevas n'avaient pour but que d'inspirer aux enseignants du premier degré des leçons adaptées aux conditions locales, de susciter les propres réflexions des élèves sur ce problème et nous avions prévu des concours que nous jugions attrayants, l'ensemble ayant d'ailleurs été élaboré avec des membres de l'Enseignement. Fe fus gentiment éconduit, sous le prétexte que les programmes étaient déjà trop chargés. Ainsi apparaît-il combien, dans nos régions où la société dite de consommation n'estime la valeur d'un produit qu'en raison de son prix, le jugement peut être faussé.

Bien que les prix de l'eau de distribution soient en France, sensiblement plus élevés que dans plusieurs pays étrangers, la valeur monétaire des eaux est encore assez basse, par rapport à celle des autres substances dont nous avons besoin, pour masquer la réalité : la valeur réelle de l'eau propre et sa carence sur le plan mondial. De plus, fréquemment, les utilisateurs ne paient pas eux-mêmes directement l'eau qu'ils consomment mais 


\section{ÉDITORIAL}

au sein d'une communauté, si bien qu'ils ont l'impression d'acquitter un droit d'utilisation de l'eau, beaucoup plus que la consommation même de l'eau. Enfin, les tarifs sont dégressifs, c'est-à-dire que plus nous gaspillons l'eau, moins cher nous la pavons. C'est une erreur profonde.

Les hygiénistes ont déterminé quelle était la valeur optimale de la consommation d'eau par habitant. Ce qui apparaîtrait logique, c'est que les tarifs soient dégressifs jusqu'à cette valeur optimale et progressifs ensuite. Cette mesure rationnelle aurait le grand avantage de limiter le gaspillage des eaux. Ce qu'il est intéressant de souligner, c'est que cette méconnaissance de la valeur de l'eau est symptomatique d'une conception fausse de notre société citadine qui confond valeur et coût. Il me faut dire, cependant qu'à une toute récente réunion d'experts discutant de l'alimentation en eau de la région parisienne, j'ai appris que la consommation -n eau avait diminué par rapport aux années précédentes et certuins experts seront tentés de tirer de ce fait la conclusion que les hommes de cette région acquièrent un comportement plus raisonnable, qu'ils deciennent économes de l'eau. Fe souhaite que cette interprétation soit exacte mais nous ne devons pas oublier d'une part que le mois d'août 1977 a été particulièrement pluvieux si bien qu'on a certainement beaucoup moins arrosé son jardin et d'autre part qu'une certaine récession industrielle s'est manifestée dans la région parisienne. Les années à venir nous diront si l'interprétation première du phénomène observé est exacte, mais en attendant, nous devons continuer à alerter l'opinion de la nécessité de limiter tout gaspillage d'eau.

Pour apprécier correctement la valeur de l'eau dans l'avenir, il faut évoquer la prospective de son utilisation pour la mise en auvre d'une énergie nouvelle, celle de l'hydrogène. En effet, l'hydrogène de l'eau, libéré, constitue avec l'oxygène de l'air, un système chimique éminemment instable, susceptible de dégager une quantité considérable d'énergie en conduisant précisément à l'élément naturel qui fait l'objet de ces journées : l'eau. Cette énergie de combustion de l'hydrogène qui résulte de la liaison $\mathrm{H}$-OH fait de l'hydrogène le meilleur combustible au poids avec un pouvoir calorifique trois fois supérieur à celui des hydrocarbures, 4,5 fois supérieur au charbon. Un tel combustible est infiniment recyclable puisqu'il restitue l'eau qui lui a donné naissance. Mais précisément, cette affinité de l'hydrogène pour l'oxygène pose les difficultés de sa production. Il faut, par apport d'énergie, dissocier l'eau en hydrogène et oxygène. Ce n'est pas le lieu d'entrer ici dans les divers procédés proposés qui ont été passés en revue récemment par $\mathrm{M}$. BALACEANU et qui donnent un espoir très sérieux à l'avènement plus ou moins rapide de la civilisation qui ferait de l'eau, non seulement l'une des sources essentielles de la santé des hommes, mais de leur progrès technologique et de leur confort.

Les eaux sont indispensables à l'homme non seulement en elles-mêmes, parce qu'elles sont directement indispensables à sa physiologie, mais par les biocénoses extrêmement variées dont elles permettent l'implantation et le développement. Les biocénoses aquatiques sont très diverses et très riches. Elles assurent la vie des hommes par l'énorme masse d'oxygène produite par le phytoplancton - spécialement par le phytoplancton océanique et par la quantité et la variété des aliments produits. Car l'homme a non seulement besoin d'une certaine quantité de nourriture, mais aussi d'une alimentation variée. L'une et l'autre, il ne pourrait les obtenir s'il n'avait à sa disposition les biocénoses aquatiques. Toutefois, ces biocénoses sont fragiles. Même un changement de milieu ne résultant de l'introduction d'aucun élément toxique, mais simplement d'une action mécanique des hommes ou de la nature (constructions portuaires par exemple ou forte houle marine) peut entrainer des altérations, des destructions de coraux par exemple, qui peuvent être l'origine d'un changement de la biocénose dangereux pour l'homme. Fe prendrai pour exemple celui de la ciguatera, maladie fréquente dans les zones tropicales peuplées de récifs coralliens. Cette maladie apparaît par poussée dans certaines régions où, le plus souvent il y a eu mort de coraux, car, sur ces débris coralliens, se développent une microfaune et une microflore complexes. Des poissons et des mollusques détritivores qui consomment ces recouvrements biodétritiques deviennent toxiques. Les auteurs américains pensaient que l'agent toxicigène était une Cyanophycée. Des auteurs français travaillant en Polynésie française à l'Institut de Recherches médicales Louis Malardé de Papeete viennent d'apporter des arguments très impressionnants en faveur de l'opinion selon laquelle l'agent porteur initial de deux toxines en cause dans la maladie, la ciguatératoxine et la maïtotoxine, serait un Péridinien, le Dipsopsalis. Quoi qu'il en soit, vous voyez qu'une seule atteinte des coraux par un mouvement anormalement violent des eaux (tempête, forte houle, interventions humaines) peut entraïner une maladie humaine. A fortiori l'introduction de certains altéragènes, même en faible pourcentage, peut perturber l'équilibre des biocénoses aquatiques et avoir les plus tragiques conséquences sur la vie des hommes. Là, les exemples sont multiples et extrêmement variés. C'est pourquoi, devant les méfaits connus et les menaces de la pollution des eaux, on tente d'organiser un système mondial de surveillance. C'est le devoir de tous - et en particulier des pharmaciens-. de coopérer à cette surveillance, qui demande un effort considérable non seulement des scientifiques spécialement affectés à ce type de surveillance, mais de tous les hommes compétents de bonne volonté. Car cette surveillance n'appelle pas seulement un travail de bactériologiste et de chimiste, pour la raison très simple que si les effets nocifs de certaines eaux riches en bactéries pathogènes ou en certaines substances chimiques nocives sont actuellement bien connues et que des techniques appropriées pour les détecter ont puêtre élaborées, d'autres sont encore à découvrir et que chaque année le génie chimique met en circulation dans la nature des milliers de substances nouvelles dont les effets sur les écosystèmes aquatiques sont complètement inconnus. Il faut donc 


\section{ÉDITORIAL}

que tout homme cultivé, que tout naturaliste - et les pharmaciens sont des naturalistes - signale aux autorités compétentes toute perturbation apparaissant dans les écosystèmes aquatiques et que les corrélations envisagées avec telle ou telle activité humaine, tel ou tel phénomène naturel suscitent des recherches permettant de les confirmer ou de les infirmer. Les pharmaciens d'officine qui, du fait de leur profession, possèdent dans nos campagnes ou sur nos rivages une stabilité relative et peuvent donc ainsi constater au cours des ans l'évolution de certaines biocénoses, qui, du fait de leur formation scientifique, possèdent des qualités d'observateurs, de biologistes, de chimistes et d'hygiénistes, doivent apporter un concours très précieux à ceux qui, en trop petit nombre, ont la responsabilité de la surveillance et de la protection des eaux.

Les biocénoses aquatiques portent en elles-mêmes des collaborateurs précieux pour cette surveillance. Ily a d'abord, pour les chimistes, certains organismes vivants des règnes végétal et animal qui ont la propriété de concentrer certains polluants et qui permettent ainsi de les dépister beaucoup plus aisément que quand on les recherche dans les eaux, ou même qui constituent les seuls moyens de les mettre en évidence. Telles sont par exemple les moules, qui concentrent considérablement les substances cancérigènes. Ce sont en effet deux espèces de Moules qu'ont choisies des chercheurs de l'Institut du Cancer de Vancouver, Dunn et Stitch, qui ont apporté les résultats de leurs déterminations du benzopyrène (cancérigène puissant) chez les Mytilus edulis et les Mytilus californianus des eaux côtières de la Colombie britannique. Ces auteurs trouvent des teneurs en benzopyrène nulles ou inférieures à $0,2 \mu \mathrm{g} / \mathrm{kg}$ chez les moules récoltées dans les zones éloignées des régions habitées. Ils obtiennent des valeurs de 2 à $4 \mu \mathrm{g}$ dans l'avant-port de Vancouver et cette teneur atteint plus de $60 \mu \mathrm{g}$ près des ports de plaisance. Ce travail a eu le mérite entre autres de montrer le grand danger d'une construction des ports de plaisance situés à proximité des moulières naturelles exploitées ou des stations de mytiliculture. Il y a de plus les êtres vivants dont la sensibilité à tel ou tel altéragène est si grande qu'ils peuvent, par une mort plus ou moins rapide, servir de tests. Mais il y a surtout ceux dont la modification d'un comportement ou d'une fonction physiologique permet de dépister un polluant à ses concentrations subléthales. Ainsi les biocénoses aquatiques comportent en elles-mêmes de véritables vigies capables de donner l'alerte en cas de danger si nous savons bien les interroger : observer leur comportement ou rechercher dans leurs tissus certains composés chimiques par les méthodes analytiques les plus sûres.

Altérer les eaux, ce n'est pas seulement menacer gravement la condition physique des hommes, c'est tuer la joie de vivre, car la santé des hommes, ce n'est pas seulement la satisfaction des besoins de la machine humaine, c'est aussi la sérénité de l'âme. Déjà Hippocrate déclarait que les eaux, les airs et les lieux influencent le moral même de l'homme. Actuellement, et de plus en plus, devant les atteintes portées à l'air que nous respirons, à l'eau que nous buvons, à la qualité de nombreux aliments, l'homme s'enfonce dans un climat d'anxiété. Sans doute, s'offrent à nous, de plus en plus variés, les anxiolytiques. Remèdes précieux pour passer un cap difficile, et il ne faut pas en sous-estimer l'intérêt. Remèdes inapplicables d'une façon permanente et à tous les hommes qui méditent sur l'avenir de leurs biocénoses et qui s'inquiètent. Et pourtant, il est bien connu que l'eau claire des ruisseaux, le cours lent des rivières, la surface calme d'un étang possèdent une extraordinaire puissance d'apaisement. Le philosophe BAchelaRd, qui était né dans un pays de ruisseaux et de rivières, écrivait : "J゙e retrouve toujours la même mélancolie devant les eaux dormantes, une mélancolie très spéciale qui a la couleur d'une mare dans une forêt humide, une mêlancolie sans oppression, songeuse, lente, calme ». Et encore : "Fe ne puis m'asseoir près d'un ruisseau sans tomber dans une rêverie profonde, sans revoir mon bonheur ». En présence d'eaux tempêtueuses, au contraire, l'homme réagira de façon différente selon son tempérament, selon qu'il est sur le rivage ou sur un navire et, dans ce dernier cas, selon qu'il est responsable de celui-ci ou simple passager. Ses réactions iront de l'abattement à la lutte, de l'abandon à l'exaltation de la vie. Sans doute ne pourra-t-il se livrer à cette mélancolie calme ou à cette rêverie profonde que ressent ou dans laquelle glisse BACHelarD près des eaux tranquilles, mais dans bien des cas, la tempête agit comme un stimulant de certaines fonctions vitales.

Les eaux influent peut-être aussi sur la condition des hommes par les mouvements rythmiques du flux ou du reflux. Cette idée est déjà émise par Aristote et par Pline qui soulignent que, sur les côtes des mers à marées, un rapport existe entre le mouvement des marées et la vie des hommes.

Un vieil adage français constate :

Qui doit mourir bientôt

Etale au flot

Mais au jusant

La mort le prend.

Et Dickens, dans "David Copperfield», nous décrit la mort de Barkis à Yarmouth parmi les pêcheurs : «Il s'en ira avec la marée, dit M. Pegotty. On ne peut mourir sur les côtes qu'à marée basse comme on ne peut venir au monde qu'à marée montante et l'on n'est vraiment en pleine vie qu'à l'heure où la marée fait son plein. Lui s'en va avec la marée ». Nous restions à le regarder. Les heures passèrent. M. Pegotty me toucha le bras en me disant à voix basse, d'un air convaincu : «La marée baisse, il s'en va ». La mer acheva de baisser, il s'en alla avec la marée... ". 


\section{EDITORIAL}

Certes nous ne pourrons être fixés sur la validité de cette opinion que lorsque des études statistiques sérieuses et très étendues dans l'espace et dans le temps auront été faites sur la répartition des décès sur les côtes des mers à marée en fonction du flux et du reflux. Je n'ai pas eu connaissance de tels travaux et s'ils existent, je serais très reconnaissant aux auditeurs en ayant été informés de me les signaler.

La couleur et la transparence des eaux exercent aussi une influence certaine sur notre psychisme. Les eaux troubles d'un estuaire ou d'une mangrove n'apportent point la sérénité des eaux étonnamment limpides de certaines zones de la mer des Caraïbes. L'eau est alors source d'imagination et de poésie, non seulement par elle-même, mais par la vie qu'elle héberge. On lit, par exemple, dans le prélude de Wordsworth : «Celui qui se penche par-dessus le bord d'une barque lente, sur le sein d'une eau tranquille, se plaisant aux découvertes que fait son oil au fond des eaux, voit mille choses belles, des herbes, des grottes, des galets, et en imagine plus encore "... C'est vrai, $j$ 'ai eu parfois l'impression de franchir les frontières du réel en admirant certains sites sous-marins d'Océanie ou des Bahamas, mais il suffit de quelques boîtes de conserve ou d'un vieux soulier pour que le charme soit rompu et que disparaisse l'impression que, selon l'expression de BAGHELARD, "l'eau fabrique de la beauté ».

C'est aussi par leur silence ou leurs chants que les eaux modèlent nos sentiments. Le rire des ruisseaux ironiques, la gaieté bruyante des cascades, le fracas des tempêtes nous inspirent des états d'âme différents.

Les eaux portent les odeurs des écosystèmes qu'elles abritent ou qu'elles humectent. Qui de nous n'a senti disparaître des anxiétés nocturnes à l'odeur de la rosée d'un matin. J' ai ressenti une vague d'odeurs marines comme une aile qui m'emportait hors du temps. Peut-être est-ce la réminiscence obscure, organique de cette soupe marine à laquelle je faisais allusion il y a un instant et qui est supposée se trouver à l'origine de la vie, qui inspire cette sensation de partir hors des limites temporelles humaines. En fait, il n'est pas un de nos sens qui ne soit sensible à certains caractères des eaux et par lequel celui-ci touche notre psychisme.

Et voilà certaines des raisons pour lesquelles, de plus en plus, les hommes se pressent sur les bords des rivières, des lacs, des mers, des lagons. Mais en s'agglutinant sur les rives ou les rivages avant d'avoir pris les mesures nécessaires à l'épuration de leurs déchets domestiques ou industriels, en se ruant sur les écosystèmes pour exploiter, souvent sans aucune mesure, certaines de leurs richesses (la récolte abusive des coquillages, par exemple) les hommes ruinent les merveilles qui les avaient attirés. Il n'est besoin d'être ni bactériologiste, ni chimiste, ni naturaliste pour s'en rendre compte. Si vous faites en bateau, et par beau temps, le tour de l'île de Tahiti, regardant les eaux à l'étrave du navire, vous pouvez, chaque fois que vous passez devant la passe d'un lagon, dire, à la simple vue des eaux, sans même regarder le rivage, si la population y est dense ou clairsemée. La situation est comparable sur les bords de certains lacs.

En fait, la santé des eaux dépend en grande partie de la clairvoyance et de la sagesse de tous les hommes. Les autorités gouvernementales connaissent maintenant les dangers qui menacent les hommes en matière d'eaux douces et marines, et des organismes ont été créés pour lutter contre ces dangers. Mais les moyens de lutte sont onéreux et l'électeur n'est souvent pas disposé à sacrifier aux entreprises nécessaires pour maintenir ou reconstituer des eaux pures, son tiercé ou son loto, son apéritif ou ses cigarettes; c'est une aberration, mais c'est ainsi.

De plus, à côté des pollutions limitées dans l'espace, qui relèvent des autorités locales ou nationales, il y a toute une série de polluants qui sont véhiculés fort loin de leur point d'origine par les aérosols, les courants aériens et aquatiques, les êtres vivants pélagiques qu'ils comportent et les animaux migrateurs, et la lutte doit être alors organisée sur le plan mondial. Cette diffusion des polluants sur toute la surface de notre planète a été bien mise en évidence lors des explosions nucléaires expérimentales en milieu aérien qui ont libéré non seulement de gros débris activés qui retombent en quelques heures, essentiellement sous l'effet de la pesanteur, dans un rayon de quelques dizaines, ou au plus de quelques centaines de kilomètres, mais des retombées lointaines constituées de particules plus fines projetées dans les basses couches de l'atmosphère (jusqu'à $12000 \mathrm{~m}$ en moyenne), qui sont transportées fort loin par les vents dominants et qui retombent peu à peu, souvent ramenées au sol par les précipitations atmosphériques, mais pour une bonne part à des milliers de kilomètres des lieux d'origine. Enfin, il y a les retombées mondiales qui proviennent des particules les plus fines atteignant la stratosphère (au-dessus de $12.000 \mathrm{~m}$ ) et sur lesquelles l'effet de la pesanteur est très faible. Mais depuis, on a montré que bien d'autres polluants (organiques comme le DDT, minéraux comme le plomb) sont susceptibles d'ètre diffusés mondialement. D'autre part, le cycle de l'eau dans la nature, trop connu pour que je le rappelle $i c i$, avec les lointains déplacements des nuages résultant de l'évaporation fait que les ressources en eau de la planète sont très mobiles. La conclusion, c'est que tant du point de vue quantitatif que du point de vue qualitatif, le problème des eaux est un problème mondial.

Le problème de l'eau de boisson, de l'eau nécessaire à des conditions d'hygiène satisfaisantes, de l'eau d'irrigation indispensable aux cultures et donc à notre alimentation n'est en effet pas seulement un problème de qualité, mais est aussi un problème de quantité. A première vue, cette opinion peut sembler extravagante tant la masse d'eau qui se trouve à la surface de notre globe est considérable. En effet, les experts admettent qu'il y a environ 17 milliards 400 millions de $\mathrm{km}^{3}$ d'eau sur la terre mais $97,3 \%$ sont salés et donc inutili- 


\section{EDITORIAL}

sables directement pour atteindre les buts qui viennent d'être évoqués. Sans doute dispose-t-on aujourd'hui de divers procédés, mais qui sont onéreux, encore que des progrès aient été réalisés dans le sens d'un moindre coût, pour obtenir la désalinisation de ces eaux. L'eau douce ne compte que pour 2,7\% dans notre hydrosphère mais la presque totalité en est bloquée sous forme de glace dans les calottes polaires ou enfouies dans les profondeurs de la terre où on peut sans doute aller en chercher une partie, mais au prix de travaux coûteux. De plus en ce qui concerne les pays dits développés, la plupart des nappes aisément exploitables sont en service et certaines d'entre elles montrent des signes évidents d'épuisement. La situation est donc préoccupante et d'autant plus que les besoins en eau de l'humanité augmentent rapidement du fait de l'accroissement de la population mondiale et de l'accession à de meilleures conditions d'hygiène des populations des pays en voie de développement, du fait aussi que l'accroissement de cette population mondiale impose à celle-ci de peupler des espaces désertiques et d'accroître ses besoins en eau d'irrigation, du fait enfin que la superficie des déserts a sensiblement augmenté au cours de ces dernières décennies, phénomène qui a suscité la toute récente conférence des Nations Unies de Nairobi. Il faut donc attaquer mondialement le problème des eaux et il l'est effectivement, mais cela ne dispense pas chaque pays, chaque région des efforts qui, même localisés, seront payants, qui seront exemplaires et qui sont indispensables pour permettre ultérieurement des solutions mondialistes. C'est par la multiplication d'efforts parcellaires, nationaux, départementaux, municipaux, plus ou moins localisés à des cours d'eau, à des rivages, que nous arriverons à une solution nationale satisfaisante de ces problèmes, solution à laquelle travaillent déjà diverses entreprises et notamment les agences de bassin et le Conservatoire du Littoral. Mais c'est par des ententes entre plusieurs états riverains d'un même fleuve ou d'une même mer que nous préparerons les voies à une protection mondiale des eaux.

Nos devoirs à l'égard des pays en voie de développement sont particulièrement impérieux. Il nous faut leur montrer la voie qui les incite à s'engager dans une technologie qui leur évite les erreurs qui ont été commises par tant de pays dits développés et qui ont conduit ceux-ci à des conditions actuelles critiques dont ils éprouvent les plus grandes difficultés à se libérer. Ainsi par exemple serait-il raisonnable, pour une ville qui naît, d'installer dès le départ un dispositif de séparation des substances organiques qui permette la vente de compost et de vieux métaux, système pratiqué avec succès et profit en Hollande et en Union Soviétique depuis un demi siècle, de mettre en auvre les agencements qui rendent possible la récupération "des déchets organiques par transformation en protéines par des organismes unicellulaires qui, mélangés à de la paille hachée, donnent des aliments vendus aux éleveurs par les marchands de fourrage. On a calculé que le profit de la vente de ces aliments produits à partir des eaux usées d'une ville de 250.000 habitants peut atteindre près d'un million de dollars par an. Ce sont là des dispositions qui permettent de rentabiliser les investissements entrepris pour épurer les eaux domestiques.

Deux centres collaborateurs de l'O.M.S., celui de Leidschendam aux Pays-Bas et celui du lac Ontario au Canada, contribuent efficacement à la création de systèmes de surveillance et de distribution des eaux dans les pays en voie de développement. Ces centres ne se considèrent pas comme devant réaliser eux-mêmes les installations nécessaires aux dits pay's, mais comme des éléments moteurs chargés de faire démarrer les opérations nécessaires sur de bons rails, de former les spécialistes nécessaires dans la population elle-même et devant s'effacer dès que l'aide n'est plus nécessaire. Cette coopération des hommes de divers pays pour une amélioration des conditions de vie dans des régions très éloignées, pour un idéal d'un mieux être répond heureusement à ce sage conseil que donnait SAINT-EXUPÉRY : «Force-les de construire une tour et tu en feras des frères». Il n'est pas question, bien entendu, de "forcer les hommes", mais de les inciter et pour ma part, en voyant s'élaborer des ouvres collectives dans les pays du Tiers Monde grâce à une collaboration internationale, $j$ 'ai souvent pensé à cette tour de SAINT-EXUPÉRY qui peut aussi bien représenter un château d'eau que le château de nos rêves, de nos rêves d'une fraternité mondiale.

Pour assurer l'efficacité de ces efforts que beaucoup d'entre nous ont entrepris avec le plus grand enthousiasme, mais avec une claire vision des difficultés immenses à vaincre, nous vous demandons respectueusement, Madame le Ministre, de continuer à nous aider, car la santé et la fraternité des hommes passent inéluctablement par la santé des eaux. Nous voudrions redonner à notre peuple jardinier qu'évoquait Péguy, non seulement, et selon sa propre expression, "le goût de faire des eaux corrompues de beaux ruisselets d'eaux vives ", mais la volonté d'accomplir l'effort nécessaire pour obtenir ce résultat en France mème et pour le susciter et l'appuyer dans le monde. Car la pureté retrouvée ou préservée d'une partie importante des eaux ne résultera pas seulement d'interdictions mais de réalisations exigeant énergie et civisme, ce terme représentant ici la qualité non seulement d'un membre d'une nation, mais d'un citoyen du monde. Demander un tel effort en ce temps où il est jugé généralement plus opportun de ne parler aux citoyens que de réduction de temps de travail, de retraite anticipée, de loisirs, de jouissance immédiate ou à court terme, paraît vraiment incongru, mais je pense que plus que jamais l'homme devrait trouver son bonheur dans un travail qui vise à laisser aux générations futures une hydrosphère en voie de régénération et que lorsque des peuples ont su trouver l'énergie nécessaire pour se battre avec un magnifique courage et un admirable esprit de sacrifice pendant des guerres fameuses, mais déplorables, ils doivent trouver celle qui est indispensable pour gagner un combat non moins impérieux, mais qui cimente la solidarité de tous les hommes : le combat pacifique, universel et fraternel, des eaux pures. 